\title{
Psicooncología
}

ISSN: 1696-7240

\section{Tecnologías de Información y Comunicación (TIC) aplicadas en cuida- dos paliativos: revisión de tema}

\author{
Verónica A. Alvarez-Tobón ${ }^{1, *}$; Ivan F. Luna-Gómez²; Ever A. Torres-Silva ${ }^{3}$; Andrea Higuita- \\ Úsuga $^{4}$; Paula T. Rivera-Mejía ${ }^{5}$
}

Recibido: 18 de diciembre de 2017 / Aceptado: 20 de agosto de 2018

Resumen: Objetivo: Revisar las herramientas utilizadas en las investigaciones de e-salud en cuidados paliativos durante los últimos cinco años Método: se realizó una revisión bibliográfica en las bases de datos PubMed, Scopus y Bireme sobre el tema de cuidados paliativos, e-salud y costos en los últimos cinco años. Resultados: El uso de las tecnologías de la información es favorable para usuarios que reciben cuidados paliativos, ya que facilita su seguimiento y el manejo de sus síntomas, además de mejorar la comunicación entre profesionales y pacientes, disminuyendo en algunos casos los desplazamientos geográficos y reduciendo los costos asociados a la atención de estos pacientes. Conclusión: la e-salud implementada a través de diferentes herramientas de intervención ha demostrado ser valiosa para los pacientes con enfermedad avanzada en cuidados paliativos, ofreciendo potenciales beneficios educativos y de optimización de recursos y tiempo para pacientes, cuidadores, que les permite mejorar su contacto con profesionales de la salud. Palabras Clave: Cuidados paliativos; cuidadores; tecnología de la información; difusión por la web.

\section{[en] Information and Communication Technologies (ICT) applied in palliative care: topic review}

\begin{abstract}
Aim: To check the tools used on the e - health researches on the palliative care item on the five last years. Method: A bibliographic review was carried out in PubMed, Scopus and Bireme databases on the subject of palliative care, e-health and costs. Results: The use of the Information technologies, the majority of the surveys find favorably the use of technology in the users who receive palliative care, since it facilitates the evaluation and handling symptoms. It improves too the communication between patients and health professionals, reducing in some cases de geographic displacements and decreasing the associated cost to the attention of these patients. Conclusion: E-health implementation through different intervention models has proven to be valuable for individuals with advance disease in palliative care, offering potential educational and the optimization of time and means for patients and caregivers, which improve the contact with the health professionals.
\end{abstract}

Keywords: Palliative care; careers; information technology; webcast.

1 Verónica A. Alvarez-Tobón. Clínica Universitaria Bolivariana, Medellín, Antioquia, Colombia

E-mail: veronicaa.alvarez@upb.edu.co-valvareztobon@gmail.com

2 Ivan F. Luna-Gómez. Universidad Pontificia Bolivariana, Medellín, Antioquia, Colombia

E-mail: ivan.luna@upb.edu.co

3 Ever A. Torres-Silva. Universidad Pontificia Bolivariana, Medellín, Antioquia, Colombia

E-mail: ever.torres@upb.edu.co

4 Andrea Higuita-Úsuga Universidad Pontificia Bolivariana, Medellín, Antioquia, Colombia

E-mail: andreahiguita17@hotmail.com

5 Paula T. Rivera- Mejía. Universidad de Caldas, Manizales, Caldas, Colombia

E-mail: paula.riveram@ucaldas.edu.co

* Dirección de correspondencia: Verónica Andrea Alvarez Tobón. Cra 72A \#78b -50, Clínica Universitaria Bolivariana, Medellín, Antioquia, Colombia. E-mail: valvareztobon@gmail.com 
Sumario. 1. Introducción 2. Planteamiento del problema 3. Métodos 3.1. Identificación de las publicaciones 4. Desarrollo del tema 4.1. Telefonía convencional 4.2. Videoconferencia 4.3. Celulares inteligentes y aplicativos móviles 4.4. Web 4.5. Ética y e-health 5. Discusión 6. Conclusiones 7. Referencias Bibliográficas.

Cómo citar: Alvarez-Tobón VA, Luna-Gómez IF, Torres-Silva EA, Higuita-Úsuga A, Rivera-Mejía PT. Tecnologías de información y comunicación (TIC) aplicadas en cuidados paliativos: revisión de tema. Psicooncología 2018;15:345-360. doi: 10.5209/PSIC.61440.

\section{Introducción}

La Organización Mundial de la Salud (OMS) define la telemedicina como "la prestación de servicios de salud como: el uso de las tecnologías informáticas para el intercambio de información válida para el diagnóstico, tratamiento y prevención de enfermedades y heridas, investigación y evaluación y para la educación continua de los proveedores, en donde la distancia es un factor crítico por todos los profesionales de la salud, en beneficio de la promoción de la salud de los individuos y sus comunidades". En este sentido, el monitoreo de los pacientes a través de las tecnologías de la información y la comunicación (TIC) es llamado telecuidado o telesalud y se ha evidenciado un incremento en su demanda ${ }^{(1)}$. Este concepto se basa en una relación proveedor-paciente y paciente-proveedor que utiliza herramientas tecnológicas de sonido, video, texto e imágenes e incluye la asistencia a distancia, la educación en salud, la investigación y la administración.

Por su parte, la Telesalud se define como "el conjunto de actividades relacionadas con la salud, servicios y métodos, los cuales se llevan a cabo a distancia con las tecnologías de información y telecomunicación, e incluye, entre otras, la telemedicina y la teleeducación en salud"(2). De igual forma, se ha definido como la prestación a distancia de servicios de salud, relacionados con la promoción, prevención, diagnóstico, tratamiento y rehabilitación, realizada por profesionales de la salud que utilizan tecnologías informáticas y de comunicación, que les permiten intercambiar información para facilitar el acceso y la prestación de servicios de salud oportunos a la población que presenta limitaciones de oferta, de acceso a los servicios o de ambos en su localización geográfica ${ }^{(3)}$.

El observatorio mundial de la salud define mHealth o salud móvil como práctica médica y de salud pública apoyada por dispositivos móviles como celulares, dispositivos de monitoreo de pacientes, asistentes digitales personales (PDA) y otros dispositivos inalámbricos ${ }^{(4)}$, la cual tiene como beneficios la atención continua de los pacientes con enfermedad avanzada en el hogar con la ayuda del cuidador que pueden llevarse a cabo a través de aplicaciones de teléfonos inteligentes que puedan mejorar esta modalidad.

Los anteriores conceptos se han desarrollado a través del área de estudio de la informática médica, la cual es definida como la aplicación de la ciencia de la información como datos más significativos para los problemas de interés biomédico ${ }^{(5)}$.

Es importante definir que los cuidados paliativos (CP) se constituyen como la atención dirigida a pacientes y cuidadores cuyo propósito es mejorar la calidad de vida, específicamente cuando afrontan problemas inherentes a una enfermedad 
potencialmente mortal o un dolor crónico. Tienen como objetivo controlar los síntomas en la etapa de la enfermedad y apoyar a los pacientes a reducir el sufrimiento, el de sus familiares y mejorar la calidad de vida hasta el final de ésta ${ }^{(6)}$, por estos motivos el objetivo de esta revisión fue revisar las herramientas utilizadas en las investigaciones de e-salud en cuidados paliativos durante los últimos cinco años.

\section{Planteamiento del problema}

La Organización Mundial de la Salud (OMS) define la atención paliativa como "un enfoque que mejora la calidad de vida de los pacientes y sus familias frente a los problemas asociados con las enfermedades potencialmente mortales, mediante la prevención y el alivio del sufrimiento", además, de ser "un sistema de apoyo para ayudar a los pacientes a vivir lo más activamente posible hasta la muerte"(7). En este sentido se conoce que hay más de 20 millones de pacientes en el mundo que necesitan cuidados paliativos al final de la vida, los cuales implican un apoyo a los familiares del paciente de parte de los servicios de salud los cuales aumentarán progresivamente ${ }^{(8)}$.

Para el caso de Latinoamérica, el desarrollo de los cuidados paliativos ha sido errático y sin un patrón definido, se conoce que existen 922 servicios en toda la región, lo que significa 1,63 servicios/unidades/equipos de cuidados por 1.000 .000 de habitantes. Los CP están en diferentes etapas de desarrollo, por lo cual cada país enfrenta muchos retos y a pesar de los grandes esfuerzos de los paliativistas y algunas veces de los gobiernos, la mayoría de la población sigue estando sin cobertura en cuidados paliativos $^{(9)}$. Si bien, existen numerosas instituciones que prestan estos servicios y que aplican las directrices de la OMS, que comprenden el alivio del dolor, afirmar la vida y considerar la muerte como un proceso normal. Pero aún persisten dificultades para prestar el servicio y brindar educación a los pacientes en las regiones alejadas ${ }^{(10)}$.

No son solo los servicios de CP los que tienen la responsabilidad de cuidar a los pacientes, también están en la escena otros actores fundamentales: los cuidadores quienes en algunos casos no cuentan con la formación o la disposición necesaria para acompañar y asistir a los familiares o seres queridos que estén en dicha situación. Lo cual resalta la importancia de la formación de los cuidadores para afrontar las situaciones que generan disconfort y preocupación entorno a los cuidados paliativos. En un estudio realizado en Colombia sobre cuidadores, se describió que más del $50 \%$ de los encuestados consideró que le hace falta preparación para realizar sus actividades de cuidado y que la mayoría de las personas cuidadoras familiares (entre $60 \%$ y $90 \%$ ) no ha participado en los diferentes modos de formación o capacitación ${ }^{(11)}$. Sin embargo, a pesar de la falta de formación que estos cuidadores declaran, se ha demostrado que la participación de las familias en el cuidado mejora la probabilidad de que una persona muera en condiciones dignas y reduce la carga de síntomas asociada con una enfermedad avanzada. Situación especialmente importante dado que las acciones y procedimientos que los servicios de salud planean para estos usuarios se basan en un plazo concreto de supervivencia esperada y no en aquellas necesidades del paciente y su familia, que pueden extenderse más allá del tiempo esperado que los cuidadores acompañen a los pacientes ${ }^{(12)}$.

Sumado a esta situación, existen limitantes del acceso a los servicios de salud, tales como las barreras de tipo geográfico, económico y de infraestructura, entre 
otras. Una revisión sistemática realizada en Alemania, Perú y Chile, identificó, que los pacientes de la zona urbana tenían mayores facilidades para acceder a los cuidados paliativos comparados con los que estaban en las zonas rurales ${ }^{(13)}$.

Dadas las barreras para acceder, el déficit en la formación de cuidadores, profesionales y pacientes; y el cambio constante de los sistemas de salud que se están transformando en modelos más interactivos de cuidado y centrados en el paciente ${ }^{(14,15)}$, las TIC han tomado un rol relevante en los CP, las cuales actualmente se utilizan como herramientas que apoyan los sistemas de salud y que se enmarcan en el concepto de e-salud.

El uso de la e-salud se encuentra cada vez más extendido y es herramienta fundamental de algunas redes y países, como en la red de telesalud de las Américas ${ }^{(16,17)}$ que realiza actividades como: identificación de las mejores prácticas locales en telesalud y en e-salud de las instituciones y sus regiones, además de generar contenidos digitales en promoción de la salud. Específicamente en países de Latinoamérica como Colombia, México y Chile se ha realizado un gran despliegue de la llamada "m health" (salud móvil) que se refiere al uso de dispositivos móviles que puedan facilitar la comunicación ${ }^{(18-21)}$.

\section{Métodos}

\subsection{Identificación de las publicaciones}

La metodología comenzó con la búsqueda de artículos en inglés y español en las bases de datos PubMed, Scopus y Bireme, los términos de búsqueda utilizados fueron a través de palabras claves en MeSH y DeCS "Telemedicine AND palliative care and costs" and "Telemedicine AND Palliative care". Primero se realizó la búsqueda en las bases de datos obteniendo los resultados de acuerdo a las ecuaciones, luego se realizó el filtro por periodo de publicación de los últimos cinco años y posteriormente se procedió a la revisión de los títulos y sus respectivos resúmenes. El número de artículos y las bases de datos consultadas se muestran en la tabla 1.

Tabla 1. Estrategia de búsqueda en base de datos electrónicas.

\begin{tabular}{ll}
\hline \multicolumn{1}{c}{ Base de datos } & \multicolumn{1}{c}{ Artículos encontrados } \\
\hline PubMed: & 124 Publicaciones. \\
& $\begin{array}{l}\text { Publicaciones de } 5 \text { años hacia adelante: } 67 \\
\text { Se usaron 67 artículos }\end{array}$ \\
\hline Scopus: & 131 Publicaciones de 5 años en adelante: 71 \\
& De los cuales 35 artículos ya estaban incluidos en la búsqueda de \\
& Pubmed, por lo cual se usaron 36 artículos. \\
\hline Bireme: & 83 artículos \\
& Publicaciones de 5 años hacia adelante: 46 \\
& De los cuales solo 36 ya estaban incluidos en la búsqueda de Scopus \\
& y Pubmed, por lo cual se usaron 10 artículos. \\
\hline
\end{tabular}


Cada artículo fue revisado y consignado en una base de datos, la cual tenía los siguientes campos: título, resumen, modelo utilizado, descripción de herramientas, aspectos éticos, aspectos económicos, observaciones. A partir de este informe se extrajo la información requerida, como parte de los criterios de inclusión en la búsqueda se evaluaron los títulos y resúmenes de los estudios identificados por la estrategia de búsqueda. Para seleccionar los artículos por medio de los títulos, se tuvo en cuenta que se incluyeran las palabras: Cuidados Paliativos y Telemedicina en su correspondiente idioma. Para la selección de los resúmenes, se tuvo en cuenta que incluyeran las palabras: uso de tecnologías, modelos de telecuidado y costos en materia de cuidados paliativos. Se incluyeron los artículos con un periodo de publicación de 5 años atrás, sin discriminar el tipo de idioma, lo cual se evidencia en la figura 1.

Figura 1. Diagrama de flujo de artículos seleccionados y excluidos.

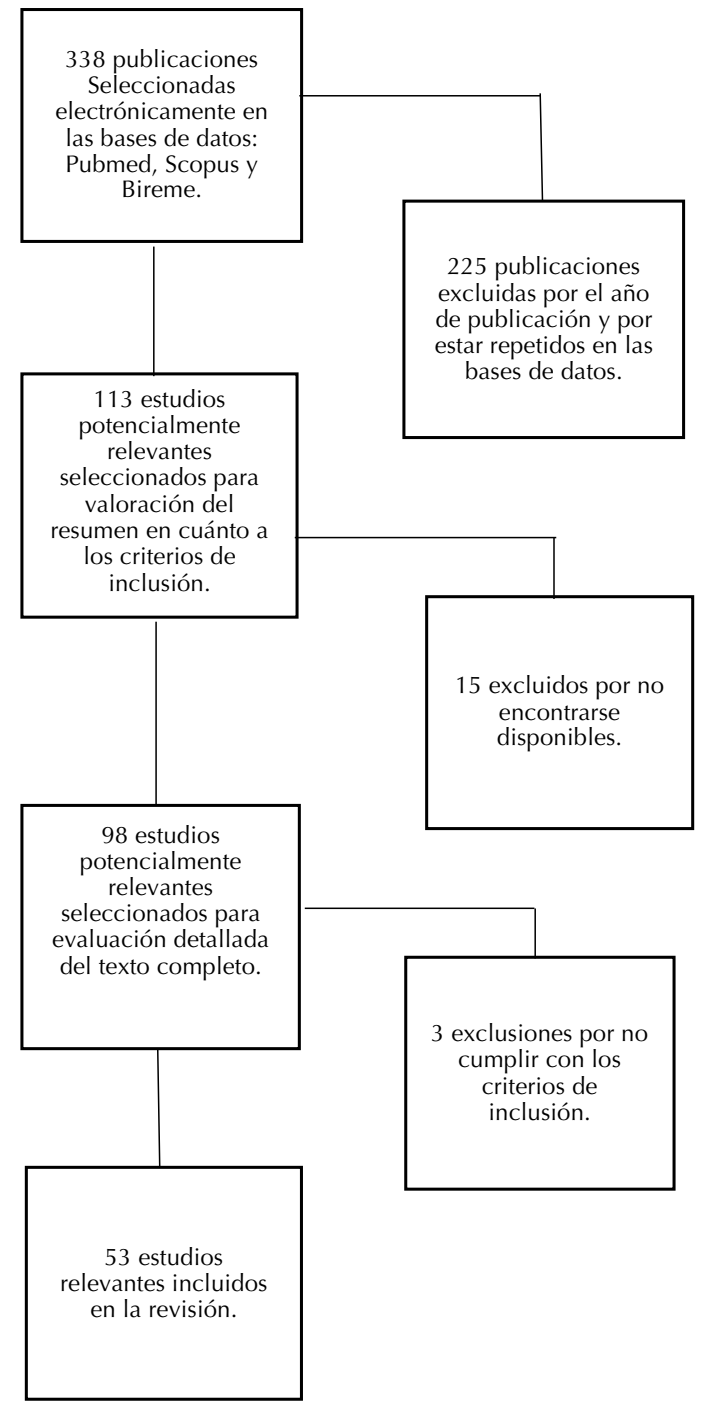




\section{Desarrollo del tema}

\subsection{Telefonía convencional}

Como parte del seguimiento a los pacientes de CP, se encuentran las llamadas telefónicas que permiten el control de síntomas derivados de esta condición. Es así como en un estudio realizado en Indiana Estados Unidos se encontró que la gestión centralizada en las llamadas por teléfono generaba mejorías significativas tanto en el dolor y la depresión de los pacientes con cáncer, en especial en las zonas rurales, en los cuales las largas distancias y los costos del desplazamiento influían en el acceso. Describiendo así las llamadas como una herramienta que genera transición entre la atención del hospital y el hogar ${ }^{(22)}$.

De otro lado en el estado de Palmerston North en Nueva Zelanda se realizó una revisión sistemática en la cual se buscaba definir la utilidad de un modelo de atención que integrara la telemedicina con la atención primaria para los pacientes de cuidados paliativos con insuficiencia cardiaca. Se llevó a cabo un seguimiento telefónico, el cual fue dirigido por el personal de enfermería, el hallazgo principal fue que las TIC ayudaron a la comunicación y a la toma de decisiones en el tratamiento de los pacientes de cuidado paliativo(16).

Por su parte Zhoun et al., en una revisión de tema, describieron el éxito de la utilización de teléfonos en el seguimiento de los pacientes de cuidados paliativos, identificaron que este método puede ayudar a reducir la carga presentada con los seguimientos clínicos y al mejoramiento de la calidad de vida, en casos de cáncer de mama, cáncer de ovario, cáncer de próstata y glioma maligno. Concluyendo así, que el monitoreo de los pacientes por medio del teléfono reduce significativamente la ansiedad y la depresión más que el seguimiento en la consulta presencial ${ }^{(17)}$.

Otro estudio fue el realizado por Verma et al., en donde se propuso una visión de la atención sanitaria centrada en el paciente con enfermedad hepática. Para ello se desarrolló un modelo a través del monitoreo remoto por medio de llamadas telefónicas provisionales. Este modelo tuvo una gran relevancia en la reducción de los costos de la atención, mejoró la calidad de la atención y la satisfacción de los pacientes ${ }^{(18)}$.

Por último, en un trabajo de investigación realizado por Davis et al., se muestra cómo un programa de intervención telefónica llamado "teleCaring" para pacientes de cuidados paliativos, el cual consistió en llamadas telefónicas proactivas diarias a pacientes y cuidadores por parte de los especialistas y enfermeras cuyo enfoque se centró en evaluar las necesidades y los síntomas de los pacientes de cuidados paliativos y brindar apoyo. Los autores describieron que su aplicación condujo a una utilización más eficiente de los recursos, la disminución de las llamadas al centro de salud después de horas y a una mayor satisfacción entre los cuidadores y aceptación de los pacientes. Las llamadas telefónicas una o dos veces por día se asociaron con una disminución de visitas del $50 \%$ al médico y al servicio de enfermería, adicionalmente los participantes de "teleCaring" tuvieron una menor utilización de los servicios clínicos, en comparación con aquellos que no lo usaron ${ }^{(23)}$.

\subsection{Videoconferencia}

En una investigación cualitativa realizada en Holanda en el año 2015 con pacientes, familiares y cuidadores, se encontró que la consulta mediada por un 
sistema de conferencias ofreció encuentros entre pacientes de cuidados paliativos domiciliarios y profesionales además de una visión única de la vida diaria de los pacientes y la interacción a largo plazo que da como resultado relaciones y experiencias de confianza, de intimidad y alivio $^{(24)}$.

En un estudio realizado por Gurp et al., en el año $2013^{(25)}$, se identificaron dos grandes hallazgos respecto a la consulta mediada por videoconferencia: 1) podría obstaculizar la proximidad física, comprometiendo la anamnesis y el diagnóstico de los pacientes con enfermedad aguda o nuevas, 2) Podría interrumpir el proceso de atención en salud y la autonomía de los médicos. En caso contrario esa herramienta tiene un efecto positivo debido a que ofrece la posibilidad de que los profesionales vean a sus pacientes desde el hogar de estos e incluso permite tratar a distancia, además les da tiempo para verificar las historias de los pacientes; De igual manera, la consulta sincrónica ayudó a los pacientes a ponerse en el centro de su propio cuidado.

En el Royal Children〉s Hospital ( $\mathrm{RCH})$ de Brisbane, se implementó un Programa de Telesalud en el Hogar, para proporcionar consultas a las familias, en el cual se señaló la consulta de vídeo en el hogar como el método más económico para proporcionar una consulta. Los mayores ahorros para el servicio de salud fueron los asociados con el tiempo que los médicos se toman para el desplazamiento. Este estudio se llevó durante un período de 24 meses, en el cual se evitaron costos significativos al Servicio de Salud Infantil ${ }^{(26)}$.

Bajo esta misma herramienta, se publicó un estudio que describió el sistema de teleconsulta experimental, llamado Telegeria HD para la atención de usuarios geriátricos, que se estableció entre el hospital geriátrico Vaugirard (VH) y el Hospital Europeo Georges Pompidou (GPEH) que utiliza una plataforma que transmite imágenes en tiempo real entre las dos instituciones. El autor refiere que esta herramienta suele ahorrar gastos derivados de los desplazamientos que por lo general duran medio día desde el centro geriátrico al hospital. Además, las consultas con los especialistas son costosas, mientras que con el uso de la herramienta la inversión es menor ${ }^{(27)}$.

Otra investigación llevada a cabo en Singapur en el año 2010, pretendía mejorar la atención en hogares de ancianos con cuidado paliativos y reducir las consultas innecesarias en el hospital mediante la instalación de un programa piloto de telemedicina. La herramienta consistió en la instalación de un sistema de banda ancha con monitores, cámaras y un sistema de micrófono, el servicio incluía consultas de telemedicina, conferencias con el familiar y reuniones con el equipo multidisciplinario, de esta manera el personal que usaba la herramienta en el centro geriátrico sentía tranquilidad y confianza ya que tenían a su disposición personal médico que guiaba y supervisaba el cuidado de los pacientes por medio de videoconferencia y la respuesta era muy rápida ${ }^{(28)}$.

Por su parte, en otro estudio realizado entre enero de 2011 y agosto de 2013 en el que monitorearon pacientes con cáncer avanzado remitidos por el Centro de Cuidados Paliativos del Hospital Universitario Pedro Ernesto en Rio de Janeiro (UERJ), estudió la viabilidad de un modelo basado en la telesalud de la prestación de servicios para los pacientes que requerían cuidados paliativos, el sistema se basaba en videoconferencias entre el personal de servicio de salud y el paciente / cuidador; este ensayo mostró que los pacientes y los cuidadores podrían administrar la tecnología y proporcionar datos que de otro modo no estarían disponibles. Las enfermeras también indicaron que la tecnología había sido efectiva para asegurar que 
el paciente o cuidador fuera tranquilizado, así mismo refirieron una identificación y manejo más rápido de los problemas y la capacidad de compartir información con otros profesionales de la salud. Los médicos informaron que la calidad del contacto de tele salud era aceptable y en la mayoría de los casos comparada con los modos de contacto actuales ${ }^{(29)}$.

En otro estudio realizado en 2014 los hallazgos confirmaron que las consultas paliativas de telemedicina son tan eficaces como las consultas cara a cara dado que los componentes de la consulta de cuidados paliativos se documentaron tal y como si hubieran ocurrido presencialmente independientemente del método de consulta ${ }^{(24)}$. Otro estudio que afianza este argumento fue uno realizado en un grupo de niños, en el cual se compararon los registros de consultas paliativas pediátricas realizadas cara a cara, con consultas de telemedicina realizadas en los hogares de los pacientes y confirmaron que las consultas paliativas a través de la telemedicina son tan efectivas como las consultas cara a cara en términos de los componentes documentados de la consulta ${ }^{(30)}$.

En cuanto al seguimiento de los pacientes en un Hogar de Atención del Centro Hospitalario Universitario de Grenoble se utilizó un sistema de videoconferencia para vincular a pacientes con cáncer que reciben quimioterapia con los proveedores de atención. Los resultados indicaron que el uso de estos dispositivos era factible y satisfactorio y que podían tener un resultado positivo en la calidad de vida del paciente con cáncer en el hogar. Otro servicio que usaban los pacientes era la confirmación de sus citas en el hospital y la interpretación de los resultados de las diferentes pruebas radiológicas realizadas por sus proveedores de atención, así como el acceso a las dosis de sus nuevos medicamentos. Al final del estudio se confirmó que el uso del sistema había mejorado los sentimientos de seguridad y las relaciones con el personal médico. Además, los resultados muestran una disminución en los niveles de ansiedad y depresión de los pacientes, así como la comprobación de la costo-efectividad de la herramienta ${ }^{(31)}$.

En un estudio realizado en población infantil, se describió que mediante la implementación de las tecnologías de la información en la atención del paciente, se evitarían costos significativos como los gastos de hospitalización, equipos e infraestructura y gastos de desplazamiento a los centros de salud, esta última planteada como una barrera para acceder a los cuidados paliativos ${ }^{(26)}$.

Un beneficio adicional de la e-salud es la reducción de costos asociada a la atención presencial, que ha confirmado los beneficios para el paciente en el CP y el uso de la tecnología en su cuidado, específicamente de la población que vive en el área rural. Aunque se ha podido evidenciar si bien puede que la relación interpersonal pueda verse afectada, se han identificado varias ventajas a nivel organizacional y mayor seguimiento a los pacientes que reducen el desplazamiento hasta los centros de atención.

\subsection{Celulares inteligentes y aplicativos móviles}

Los teléfonos inteligentes son avanzados en materia tecnológica, usan un software similar a los dispositivos computacionales usados con una interfaz amigable ${ }^{(32)}$. El uso de éstos, así como sus aplicaciones de software proveen a los profesionales de la salud las oportunidades para integrar la tecnología con la práctica clínica ${ }^{(33)}$. Las aplicaciones prácticas de estos en la vida cotidiana incluyen: servicios de mensajería, 
recordatorio de $\operatorname{citas}^{(34)}$, monitorización de condiciones médicas crónicas ${ }^{(35)}$ y el mejoramiento de la satisfacción con el tratamiento y el apoyo psicológico ${ }^{(36)}$. Además han demostrado su amplia aplicación para mejorar el diagnóstico, la eficiencia y como soporte para los programas de salud pública ${ }^{(37)}$.

Como caso específico puede citarse el uso de los celulares inteligentes que redujeron significativamente las interrupciones del paciente, además de permitir que las enfermeras permanecieran con los pacientes y redujeran el tiempo de espera para regresar una llamada ${ }^{(38)}$. Una de las aplicaciones más usadas es WhatsApp Messenger, que permite al usuario compartir imágenes clínicas, video clínico, fotografía de informes, fotografía de medicamentos y mensajes de texto puede llevar la gestión de cuidados paliativos a un nuevo nivel ${ }^{(39)}$.

Con respecto a los costos, los autores Dhiliwal y Salins describieron que en un escenario de cuidado paliativo de un programa con comunicaciones audiovisuales potenciadas por aplicaciones de celulares inteligentes, es posible disminuir la carga de los cuidadores así como el ahorro en viajes y consultas y previene las visitas innecesarias al hospital ${ }^{(39)}$.

En este sentido, los dispositivos electrónicos (celulares, computadores y tablets) se han convertido en generadores de desarrollo social y económico, además de que se reconoce su potencial para reducir las limitaciones de acceso. Específicamente los celulares que se han enfocado en el manejo del soporte de la medicación y han enlazado la comunicación con los proveedores y facilitan la conexión de cuidadores con las redes sociales que pueden ayudar a mejorar los cuidados paliativos en adultos mayores $^{(40)}$.

Mosa et al. ${ }^{(41)}$ revisaron 55 artículos que reportaron 83 aplicaciones para celulares inteligentes así: 57 aplicaciones para profesionales de la salud enfocados en el diagnóstico de la enfermedad ${ }^{(21)}$, referencias para medicamentos ${ }^{(6)}$, calculadoras

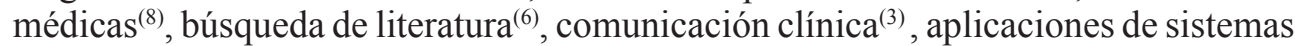
clínicos de información ${ }^{(4)}$, entrenamiento médico ${ }^{(2)}$, y aplicaciones de cuidado general $^{(7)} ; 11$ aplicaciones para estudiantes de medicina o enfermería enfocados en educación médica y 15 aplicaciones para los pacientes enfocados en el manejo de la enfermedad crónica ${ }^{(6)}$, relacionados con Enfermedades no Transmisibles $(\text { ETN })^{(4)}$, relacionados con caídas ${ }^{(3)}$ y otras dos condiciones ${ }^{(2)}$.

Con respecto al manejo del dolor a través de los celulares; Jibb et al. ${ }^{(42)}$ desarrollaron un algoritmo de decisión y un requerimiento de Sistema que informaría el manejo del dolor provisto en tiempo real por un celular inteligente para el manejo del dolor en adolescentes con cáncer. Los autores desarrollaron una revisión de literatura exhaustiva, la cual fue diseminado entre 15 expertos internacionales (clínicos, científicos y consumidores) en el dolor pediátrico, cáncer pediátrico y mHealth (salud móvil: Organización Mundial de la Salud) ${ }^{(4)}$, los expertos finalmente consensuaron recomendaciones como: "el dolor de los adolescentes requiere de una acción (dispositivo para el manejo del dolor) desde una aplicación, la cual debería proveer a los adolescentes información acerca del dolor y los requerimientos funcionales de la aplicación.

Es importante citar también las desventajas de usar los celulares, en este sentido el autor McBride ${ }^{(43)}$, reportó algunos atributos de distracción de estos dispositivos tales como: una intrusión en una tarea clínica primaria y discontinuidad de la tarea clínica. Por este motivo se sugiere que los clínicos que brindan cuidados paliativos trabajen conjuntamente con los profesionales que hacen el software para desarrollar 
aplicaciones adecuadas a las características biopsicosociales y las necesidades de la familia, los cuidadores y los pacientes ${ }^{(44)}$. Dado el auge de la portabilidad y los teléfonos celulares smartphones se vislumbra una oportunidad para el seguimiento de los pacientes como acciones de e-salud.

\subsection{Web}

En la web se encuentran portales de pacientes o aplicativos diseñados para las acciones de salud, y se han encontrado algunos beneficios del uso de la web en los $\mathrm{CP}$, tal y como lo demuestra un estudio realizado por Clarke et al, en el que los pacientes asignados a recibir una intervención de autoayuda de Internet tenían una mayor reducción en los síntomas depresivos que el grupo de control ${ }^{(7)}$.

El rápido desarrollo, adopción y uso de varias aplicaciones web presenta cambios cruciales para los clínicos y usuarios. Los desafíos en este sentido serían: asegurar la confidencialidad, seguridad, establecer la costo-efectividad y comprometer a los pacientes y profesionales a optimizar su uso en la toma de decisiones en salud. De otro lado los desafíos en el uso de las aplicaciones móviles incluyen la cobertura incompleta por la conectividad a través de las regiones, deficiencia de estándares y posible sobrecarga de información ${ }^{(45)}$.

\section{5. Ética y e- health}

El uso de TIC, plantea retos y cuestiones éticas, por lo cual los avances en este campo deben incluir beneficios para el usuario y garantizar respeto por la privacidad, dignidad y autonomía del cuidado. Como lo refiere el estudio de Chung et al, las tecnologías mejoran significativamente el cuidado, específicamente en el caso del adulto mayor, porque estos usuarios se enfrentan a un deterioro cognitivo relacionado con algunas enfermedades neurodegenerativas, en el cual su capacidad para evaluar y comprender la información puede verse comprometida y estos autores demuestran la necesidad de los adultos mayores de vivir en hogares comunicados a través de las tecnologías, es decir estar en hogares inteligentes, en los cuales su privacidad no está invadida y no son intrusivos para los demás ${ }^{(46)}$.

A pesar que se conocen opiniones favorables en torno a la utilización de modelos de atención basados en TIC para cuidados paliativos, existen algunas consideraciones en las cuales se pone de manifiesto la idea de que la implementación de las tecnologías de la información no debe convertirse en el sustituto de la evaluación médico-paciente, justificando la poca relación interpersonal en la atención médica, la cual se asume con gran importancia ${ }^{(6)}$.

Dado que la comunicación entre el personal de salud y los pacientes es un elemento fundamental para brindar información veraz y honesta a cerca del control del dolor, la muerte y un buen morir ${ }^{(47)}$, es importante tener en cuenta que la introducción de tecnologías tiene el potencial de alterar la dinámica de las relaciones entre los pacientes, sus familias y los médicos de cuidados paliativos, por lo cual es importante establecer un contacto presencial entre el paciente y el médico antes de ejercer una atención dirigida por medio de las tecnologías de la información, ya que el contacto real con el paciente no debe supeditarse solo a la teleconsulta ${ }^{(25)}$.

Existe otro aspecto desde el punto de vista ético, relacionado con el apoyo que estas herramientas tecnológicas pueden dar en el momento en el que la vida termina, 
ya que para las personas en cuidados paliativos es muy importante morir en la comodidad de su casa en medio de los recuerdos y objetos personales, teniendo en cuenta que una buena muerte es morir en paz en su propia casa. Por lo cual se ha demostrado que la telemedicina le permite al paciente tener una "buena muerte" en el hogar, a lo cual se le asigna una alta relevancia, ya que en muy pocas ocasiones las personas pueden presenciar el momento final de la muerte y estar en compañía de las personas queridas en el ámbito hospitalario ${ }^{(28,39)}$.

Según estos planteamientos, es importante tener presente que si bien la e- salud no sustituye el contacto físico con el paciente, se presenta como una herramienta que genera un puente entre el paciente, el médico y el personal de la salud.

\section{Discusión}

El objetivo de este artículo fue hacer una revisión narrativa acerca del telecuidado en los $\mathrm{CP}$, encontrándose varios estudios que concluyen que es beneficioso el uso de la tecnología en los usuarios que reciben este tipo de cuidados. Se encontró evidencia suficiente para afirmar que la tecnología es necesaria para mejorar la eficiencia y se debería incorporar en el cuidado paliativo ${ }^{(23)}$.

Las investigaciones han sido consistentes en la experiencia positiva de los pacientes con el uso de la tecnología y el mejoramiento de la comunicación entre profesionales de la salud y pacientes que permite la continuidad en el cuidado.

Relativo al tema de la tele salud y los cuidados paliativos, es necesario constatar que la intervención tiene un valor clínico directo y real para el paciente y el cuidador ${ }^{(6)}$, con respecto a la infraestructura que se debe tener para prestar los servicios de telesalud se debe tener disponibilidad de: ordenadores personales, ordenadores portátiles, clientes ligeros, smartphones, etc. ${ }^{(5)}$. Además esta atención implica una interfaz entre diferentes proveedores de servicios de salud y sistemas para ofrecer servicios, especialmente para pacientes con múltiples necesidades de cuidado ${ }^{(18)}$. En este sentido, es posible facilitar la evaluación y el manejo efectivo de síntomas reportados por los pacientes con enfermedades crónicas, mejorando la experiencia de cuidado en los pacientes ${ }^{(49)}$.

Según la revisión de artículos realizada en este estudio, la telesalud puede ser aplicada en diversidad de escenarios y por profesionales de la salud, hallazgo coherente con un artículo que encontró que la tele salud estaba siendo utilizada por una gama de profesionales de la salud en entornos de atención oncológica que incluía cuidados paliativos especializados, hospicios, centros de atención primaria, hogares de ancianos y hospitales, así como pacientes y cuidadores. La revisión sugiere que la tecnología actual es utilizable y aceptable para pacientes y profesionales de la salud en entornos de cuidados paliativos ${ }^{(30)}$.

Otro hallazgo importante extraído de la revisión es la aceptación y satisfacción de los pacientes con las TIC usadas como herramienta para sus cuidados paliativos. Lo cual está en concordancia con una investigación realizada por Owen et al, en la cual se prueban dos tecnologías de tele-salud en el hogar: "Intel Health Guide y Apple iPad", comprueba que tanto los pacientes como el personal de salud aceptan y aprenden con mayor facilidad el uso de las nuevas tecnologías de la información para el cuidado de la salud, especialmente porque su uso ahorra una cantidad significativa de tiempo ${ }^{(5)}$. 
Una ventaja interesante de la telesalud es la cobertura en salud de usuarios que residen en zonas lejanas, es allí donde la telemedicina puede asistir a una población rural cuando es parte de un modelo integrado de cuidado ${ }^{(38)}$. Esta tecnología es útil en los cuidados paliativos para pacientes que tienen dificultades en el acceso a los servicios médicos asociado al aislamiento geográfico. No obstante una investigación realizada en población del área rural refiere que se requiere más investigación en la telemonitorización antes que se puedan extraer conclusiones acerca del rol de estas tecnologías ${ }^{(16)}$.

Los pacientes que tienen enfermedades que limitan la vida pueden recibir cuidado continuo en casa con la ayuda del equipo de soporte de cuidados paliativos en casa y los médicos de familia locales. Es así como aplicaciones como "WhatsApp", permite que los usuarios compartan imágenes clínicas y video, fotografías de reportes, medicamentos y mensajes de texto que pueden llevar el manejo del cuidado paliativo en casa a un nuevo nivel al conectar los canales de comunicación entre pacientes, cuidados paliativos del médico y la familia ${ }^{(38)}$.

Otro aspecto importante a tener en cuenta en el uso de estas TIC es el balance costo beneficio, que según el investigador Johnston ${ }^{(49)}$ genera ahorro en costos económicos. Es así como una investigación llevada a cabo en pacientes con Parkinson se sugirió que debido a los crecientes costos de la hospitalización en los servicios de salud la telemedicina debe ser una opción en pacientes en estas condiciones

En este sentido, la telesalud se ha considerado como una herramienta, útil para la recuperación de la salud, la detección precoz, la prevención de daños y riesgos y la promoción de estilos de vida saludables, al disminuir los costos de desplazamiento a los centros de salud para la atención médica. lo cual se ha reportado en la literatura la eficacia de las intervenciones en salud con un balance entre costos y beneficios ${ }^{(49)}$.

A pesar de los beneficios relatados anteriormente, también existe evidencia que no es favorable en torno al tema. Es así como en una revisión sistemática de telemedicina se indica que hay una limitada evidencia alrededor de la efectividad de las intervenciones en e-salud para los cuidadores y profesionales de la salud ${ }^{(7)}$. Otro estudio argumentó que la evidencia de la aplicación de la telemedicina en cuidados paliativos es limitada especialmente en la población de adultos mayores y hay un déficit de apoyo para aplicar la telesalud en las zonas rurales de manera adecuada y segura ${ }^{(50)}$.

\section{Conclusiones}

Las acciones de salud mediadas por las TIC e implementada a través de diferentes modelos de intervención ha demostrado ser valiosa para los pacientes en cuidados paliativos, sin embargo, es importante que no se pierda el contacto "cara a cara" en su totalidad, ya que este ha resultado importante tanto para los pacientes como para los profesionales y hacer énfasis en que este tipo de alternativas son complementos y no remplazos.

Es necesario hacer un esfuerzo investigativo alrededor de la telesalud en cuidados paliativos y especialmente en adultos mayores, que permita determinar la eficacia, la medición de resultados y de costo efectividad, dado que un gran porcentaje de los pacientes que están en cuidados paliativos son adultos mayores, en este sentido, esta información resulta muy importante para establecer estrategias en el ámbito de cuidados paliativos y fortalecer las necesidades de desarrollo tecnológico en salud como un estándar de calidad en la atención de los pacientes. 


\section{Referencias Bibliográficas}

1. Brown SJ. Guest editorial: next generation telecare and its role in primary and community care. Health Soc Care Community 2003;11:459-62. doi:10.1046/j.13652524.2003.00451.x

2. Congreso de Colombia. LEY 1419 DE 2010 [Internet]. [Acceso 13 de diciembre de 2017]. Disponible en: http://www.secretariasenado.gov.co/senado/basedoc/ley/2010/ ley_1419_2010.html

3. Gómez IFL, Silva EAT, Correa CT, Zuluaga SV, Morales MMR, Serna CG, et al. Uso de las tecnologías de información y comunicación para el cuidado del binomio maternofetal: revisión de tema. Med UPB. 2016;34:138-47. doi:10.18566/medupb.v34n2.a06

4. World Health Organization. New horizons for health through mobile technologies [Internet]. [Acceso 13 de diciembre de 2017]. Disponible en: http:/www.who.int/goe/ publications/goe_mhealth_web.pdf

5. Carnicero J, Rojas D. Aplicación de las tecnologías de la información y las comunicaciones en los sistemas de salud de Bélgica, Dinamarca, España, Reino Unido y Suecia. CEPAL [Internet]. 2014 [citado 24 de abril de 2017]. Disponible en: http:/www.cepal.org/es/ publicaciones/6175-aplicacion-tecnologias-la-informacion-comunicaciones-sistemassalud-belgica

6. Tieman JJ, Morgan DD, Swetenham K, To THM, Currow DC. Designing clinically valuable telehealth resources: processes to develop a community-based palliative care prototype. JMIR Res Protoc. 4 de septiembre de 2014;3(3):e41. doi: 10.2196/resprot.3266.

7. Capurro D, Ganzinger M, Perez-Lu J, Knaup P. Effectiveness of eHealth interventions and information needs in palliative care: A systematic literature review. J Med Internet Res [Internet]. 7 de marzo de 2014 [Acceso 24 de abril de 2017];16(3). Disponible en: http://www.ncbi.nlm.nih.gov/pmc/articles/PMC3961802/

8. OMS | Primer atlas mundial de las necesidades de cuidados paliativos no atendidas [Internet]. WHO. [Acceso 22 de mayo de 2017]. Disponible en: http://www.who.int/ mediacentre/news/releases/2014/palliative-care-20140128/es/

9. Pastrana T. Atlas de cuidados paliativos en Latinoamérica. Houston; [Ansoáin, Navarra: IAHPC Press ; Errea Comunicación; 2013.

10. OPS/OMS Colombia - Las enfermedades no transmisibles (ENT), nuestro reto [Internet]. [Acceso 22 de mayo de 2017]. Disponible en: http://www.paho.org/col/index. php?option=com_content\&view=article\&id=1756:las-enfermedades-no-transmisiblesent-nuestro-reto\&Itemid $=487$

11. Ministerio de Salud y Protección Social Colciencias. Sabe Colombia 2015: Estudio nacional de salud, bienestar y envejecimiento, 2015.

12. Ministerio de Salud y Protección social. Abecé: cuidados paliativos. Bogotá (Colombia); 2016.

13. Capurro D, Ganzinger M, Pérez-Lu JE. Palliative care from a medical informatics perspective in Chile, Germany, and Peru. Stud Health Technol Inform 2013;192:1013. doi: 10.3233/978-1-61499-289-9-1013

14. Sarmiento Medina MI. El cuidado paliativo: un recurso para la atención del paciente con enfermedad terminal. Revista Salud Bosque 2011; 1:23-37 15.

15. Nasi G, Cucciniello M, Guerrazzi C. The role of mobile technologies in health care processes: the case of cancer supportive care. J Med Internet Res 2015;17(2):e26. doi: 10.2196/jmir.3757. 
16. Fernando J, Percy J, Davidson L, Allan S. The challenge of providing palliative care to a rural population with cardiovascular disease. Curr Opin Support Palliat Care 2014;8:914. doi: 10.1097/SPC.0000000000000023.

17. Zhou M, Holden L, Bedard G, Zeng L, Lam H, Chu D, et al. The utilization of telephone follow-up in the advanced cancer population: a review of the literature. J Comp Eff Res 2012;1:509-17. doi: 10.2217/cer.12.63.

18. Verma M, Navarro V. Patient-centered care: a new paradigm for chronic liver disease. Hepatol Baltim Md 2015;62:988-90. doi: 10.1002/hep.28022

19. Gonzales Retis ML, Pacheco López A. Desarrollo de la telesalud en México. Santiago de Chile; 2013.

20. Bill G, Crisci C, Canet T. La Red de Telesalud de las Américas y su papel en la atención primaria de la salud. 2014; Rev Panam Salud Publica 35(5/6).

21. Vloria Núñez C, Caballero-Uribe CV. Avances y retos para implementar la telemedicina y otras tecnologias de la informacion (TICs). Rev Científica Salud Uninorte [Internet]. 8 de mayo de 2014 [Acceso 31 de mayo de 2017]; 30 (2). Disponible en: http://rcientificas. uninorte.edu.co/index.php/salud/article/view/6641

22. Charlton M, Schlichting J, Chioreso C, Ward M, Vikas P. Challenges of rural cancer care in the United States. Oncol Williston Park 2015;29:633-40.

23. Bull J. Improving Hospice care through a proactive telephone-based quality improvement intervention: Commentary on Davis et al. J Pain Symptom Manage 2015;50:288. doi: 10.1016/j.jpainsymman.2015.07.004

24. Neergaard MA, Warfvinge JE, Jespersen TW, Olesen F, Ejskjaer N, Jensen AB. The impact of «modern telecommunication» in palliative care--the views of the professionals. Telemed J E Health 2014;20:24-31. doi: 10.1089/tmj.2013.0047

25. van Gurp J, van Selm M, van Leeuwen E, Hasselaar J. Transmural palliative care by means of teleconsultation: a window of opportunities and new restrictions. BMC Med Ethics 2013;14:12. doi: 10.1186/1472-6939-14-12.

26. Bradford NK, Armfield NR, Young J, Smith AC. Paediatric palliative care by video consultation at home: a cost minimisation analysis. BMC Health Serv Res 2014;14:328. doi: 10.1186/1472-6963-14-328.

27. Esterle L, Mathieu-FritzA. Teleconsultation in geriatrics: impact on professional practice. Int J Med Inf 2013;82:684-95. doi: 10.1016/j.ijmedinf.2013.04.006.

28. Low JA, Beins G, Lee KK, Koh E. Last moments of life: can telemedicine play a role? Palliat Support Care 2013;11:353-5. doi: 10.1017/S1478951512000995.

29. Tieman JJ, Swetenham K, Morgan DD, To TH, Currow DC. Using telehealth to support end of life care in the community: a feasibility study. BMC Palliat Care 2016;15:94. Doi: 10.1186/s12904-016-0167-7

30. Kidd L, Cayless S, Johnston B, Wengstrom Y. Telehealth in palliative care in the UK: a review of the evidence. J Telemed Telecare 2010;16:394-402. doi: 10.1258/ jtt.2010.091108

31. Mona Laila, Vincent Rialle, Lydie Nicolas, Catherine Duguay, Alain Franco. Videophones for the delivery of home healthcare in oncology - Semantic Scholar. 2008 [Acceso 31 de mayo de 2017]; Disponible en: /paper/Videophones-for-the-delivery-of-home-healthcarein-Laila-Rialle/7f74f75b8acafc4734f969247c0e6f7546a0dd85

32. Ehrler F, Walesa M, Sarrey E, Lovis C. Smartphones: Evidence-based user-interface design. Stud Health Technol Inform 2013;192:57-61. doi: 10.3233/978-1-61499-2899-57 
33. Bert F, Giacometti M, Gualano MR, Siliquini R. Smartphones and health promotion: a review of the evidence. J Med Syst 2014;38:9995. doi: 10.1007/s10916-013-9995-7

34. Bos A, Hoogstraten J, Prahl-Andersen B. Failed appointments in an orthodontic clinic. Am J Orthod Dentofacial Orthop 2005;127:355-7. doi: 10.1016/j.ajodo.2004.11.014

35. de Jongh T, Gurol-Urganci I, Vodopivec-Jamsek V, Car J, Atun R. Mobile phone messaging for facilitating self-management of long-term illnesses. Cochrane Database Syst Rev. 12 de diciembre de 2012;12:CD007459.

36. Wei J, Hollin I, Kachnowski S. A review of the use of mobile phone text messaging in clinical and healthy behaviour interventions. J Telemed Telecare 2011;17:41-8. doi: 10.1258/jtt.2010.100322.

37. da Costa TM, Salomão PL, Martha AS, Pisa IT, Sigulem D. The impact of short message service text messages sent as appointment reminders to patients' cell phones at outpatient clinics in São Paulo, Brazil. Int J Med Inf 2010;79:65-70. doi: 10.1016/j. ijmedinf.2009.09.001.

38. Whitlow ML, Drake E, Tullmann D, Hoke G, Barth D. Bringing technology to the bedside: using smartphones to improve interprofessional communication. Comput Inform Nurs CIN 2014;32:305-11. doi: 10.1097/CIN.0000000000000063.

39. Dhiliwal SR, Salins N. Smartphone applications in palliative homecare. Indian J Palliat Care 2015;21:88-91. doi: 10.4103/0973-1075.150199

40. Richardson JE, Lee JI, Nirenberg A, Reid MC. The potential role for smartphones among older adults with chronic noncancer pain: A qualitative study. Pain Med 2017; pnw284. doi: 10.1093/pm/pnw284

41. Mosa ASM, Yoo I, Sheets L. A systematic review of healthcare applications for smartphones. BMC Med Inform Decis Mak 2012;12:67. doi: 10.1186/1472-6947-12-67

42. Jibb LA, Stevens BJ, Nathan PC, Seto E, Cafazzo JA, Stinson JN. A smartphone-based pain management app for adolescents with cancer: establishing system requirements and a pain care algorithm based on literature review, interviews, and consensus. JMIR Res Protoc 2014;3(1):e15. doi: 10.2196/resprot.3041.

43. McBride DL. Distraction of clinicians by smartphones in hospitals: a concept analysis. J Adv Nurs 2015;71:2020-30. doi: 10.1111/jan.12674

44. Jamwal NR, Kumar SP. Smarter palliative care for cancer: Use of smartphone applications. Indian J Palliat Care 2016;22:108-10. doi: 10.4103/0973-1075.173950.

45. Charani E, Castro-Sánchez E, Moore LS, Holmes A. Do smartphone applications in healthcare require a governance and legal framework? It depends on the application! BMC Med 2014;12:29. doi: 10.1186/1741-7015-12-29.

46. Chung J, Demiris G, Thompson HJ. Ethical Considerations regarding the use of smart home technologies for older adults: An integrative review. Annu Rev Nurs Res 2016;34:155-81. doi: 10.1891/0739-6686.34.155.

47. van Gurp J, Hasselaar J, van Leeuwen E, Hoek P, Vissers K, van Selm M. Connecting with patients and instilling realism in an era of emerging communication possibilities: a review on palliative care communication heading to telecare practice. Patient Educ Couns 2013;93:504-14. doi: 10.1016/j.pec.2013.07.002.

48. van Gurp J, Soyannwo O, Odebunmi K, Dania S, van Selm M, van Leeuwen E, et al. Telemedicine's potential to support good dying in Nigeria: A qualitative study. PloS One. 2015;10(6):e0126820. doi: 10.1371/journal.pone.0126820 
49. Johnston B, Kidd L, Wengstrom Y, Kearney N. An evaluation of the use of Telehealth within palliative care settings across Scotland. Palliat Med. 2012;26:152-61. doi: 10.1177/0269216311398698.

50. Johnston B. UK telehealth initiatives in palliative care: a review. Int J Palliat Nurs 2011;17:301-8. doi:10.12968/ijpn.2011.17.6.301 